\title{
Relationship between Duffy Antigen Receptor for Chemokines and Clinical Characteristics in Han People with Chronic Hepatitis C Infection in Dalian, China
}

\author{
Lin-Nan Shao ${ }^{1}$, Shi-Hang Zhou ${ }^{1}$ and Ming Liu, ${ }^{2}{ }^{*}$ \\ 1 Dalian Blood Center, Dalian, Liaoning, China \\ 2 Department of Cell Biology, Dalian Medical University, Dalian, China \\ * Corresponding author: Ming Liu, Department of Cell Biology, Dalian Medical University, Dalian, China. Tel: +8641186110318; Email: \\ liuminglinxi@163.com
}

Received 2020 August 21; Revised 2020 September 11; Accepted 2020 0ctober 14.

\begin{abstract}
Background: Most patients with untreated chronic hepatitis C virus (HCV) infection develop hepatic fibrosis. Hepatic disease progression is monitored with hematological markers (alanine aminotransferase [ALT], aspartate aminotransferase [AST], albumin and platelet [PLT] count, AST/ALT ratio, AST/PLT ratio index [APRI], and fibrosis 4 score [FIB-4]) and FibroScan (manufactured in Echosens, France).

Objectives: The present study aimed to investigate the association between Duffy antigen/chemokines receptor (DARC) polymorphisms and clinical parameters in the Han people with chronic hepatitis C infection in Dalian, China.

Methods: This cohort study was performed on 245 Han people with chronic HCV at Dalian infectious hospital during April-December 2015. The participants of the research were selected using the consecutive sampling method. The DARC genotyping was performed using the TaqMan probe method and transient elastography was measured by FibroScan.

Results: Based on the findings, DARC polymorphisms correlated with ALT concentrations (FY*A/FY*A vs. FY*A/FY*B, $\mathrm{P}=0.025)$. However, the DARC polymorphism did not have an association with HCV RNA titers (FY*A/FY*A vs. FY*A/FY*B, P=0.241) or hepatic fibrosis $\left(\mathrm{FY}^{*} \mathrm{~A} / \mathrm{FY}^{*} \mathrm{~A}\right.$ vs. $\left.\mathrm{FY} * \mathrm{~A} / \mathrm{FY} * \mathrm{~B}, \mathrm{P}=0.325\right)$. Moreover, correlation analyses showed that APRI $(\mathrm{P}<0.001, \mathrm{rho}=0.603)$ and $\mathrm{FIB}-4$ $(\mathrm{P}<0.001$, rho $=0.698)$ were useful predictors of hepatic fibrosis in chronic HCV infection. Besides, HCV RNA titers $(\mathrm{P}=0.327)$ and hepatic injury markers ( $\mathrm{P}=0.814,0.198,0.767$, and 0.171 for ALT, AST, ALB, and AST/ALT, respectively) were not useful for the estimation of the fibrosis stage in patients with chronic hepatitis $\mathrm{C}$.

Conclusion: The FY*A allele is a potentially valuable protective factor against hepatocyte damage in chronic HCV-infected patients.

Keywords: DARC, HCV, Polymorphism
\end{abstract}

\section{Background}

Hepatitis $\mathrm{C}$ virus (HCV) is one of the main causes of chronic liver disease. Most chronic HCV infected individuals will develop complications, such as liver fibrosis, cirrhosis, and end-stage liver disease (1). Non-invasive methods, including some hematological markers (alanine aminotransferase [ALT], aspartate aminotransferase [AST], albumin [ALB], and platelet [PLT] count), their derived markers (AST/ALT ratio, fibrosis 4 score [FIB-4], and AST/PLT ratio index [APRI]), and transient elastography (FibroScan) were also used for monitoring fibrosis progression (2).

The Duffy antigen/chemokines receptor (DARC), also known as Duffy blood group antigens (3) or atypical chemokine receptor 1 (4), is a highly promiscuous receptor that binds a large number of chemokines $(5,6)$. The DARC gene has two major alleles in Asians, namely $F Y^{*} A$ and $F Y^{*} B$, which differ by rs12075 polymorphism (at position chr1:159 $205564 \mathrm{G}>$ A) (7). Some studies have shown that the DARC polymorphism has a critical role in Plasmodium vivax malaria, human immunodeficiency virus, and breast cancer (8-10).

Moreover, DARC has two main functions; one of which is as a decoy receptor for a variety of CXC and
CC chemokines through internalization while the other one is participation in the transportation of chemokines across endothelial cells and their presentation to leukocytes (11). The CXC and CC chemokines are believed to contribute to liver inflammation and damage during chronic HCV infection $(12,13)$. Therefore, DARC polymorphism may play a crucial role in the prevention of liver deterioration in chronic hepatitis $\mathrm{C}$.

\section{Objectives}

The primary aim of this study was to investigate the association between the DARC polymorphism and clinical characteristics in the Han people with chronic HCV infection in Dalian, China.

\section{Methods}

\subsection{Subjects}

This cohort study was performed on 245 Han patients with chronic HCV-infected who referred to Dalian infectious hospital (a Grade III Level A hospital located in northern China) from April 2015 to December 2015. The subjects were selected using the consecutive sampling method. To avoid sampling 
error and bias, the inclusion criteria were 1) the presence of HCV antibody and HCV RNA, 2) lack of ascites, 3) lack of hepatitis B virus or alcohol-related liver diseases, and 4) no significant heart, brain, lung, and kidney complications. The collected data included their age, gender, routine full blood count, liver function tests, and serum HCV RNA titers. It should be noted that the protocol was approved by the Ethics Committee of Dalian Blood Center (DBC00802007). Furthermore, informed consent was obtained from all the participants. All the collected data were anonymized to comply with the provisions of personal data protection legislation.

\subsection{DARC genotyping}

The DARC genotyping was performed by TaqMan probe method using the Applied Biosystems 7300 detection system (manufactured in CA, USA), (14). The PCR mixtures contained $10 \mu \mathrm{L} 2 \times$ TaqMan Universal PCR Master Mix (Applied Biosystems), 0.2 $\mu \mathrm{L}(20 \mu \mathrm{M})$ of each probe (FAM-AGACTATGG TGCCAAC-MGB for FY*A and VIC-AGACTATGA TGCCAACC-MGB for FY*B), $0.9 \mu \mathrm{L}(20 \mu \mathrm{M})$ of each primer (F: 5'- TGTGAATGATTCCTTCCCAGATG-3' and R: 5'- CACTGGTGAGGATGAAGAAGGG-3'), $1 \mu \mathrm{L}$ DNA, and $6.8 \mu \mathrm{L}$ distilled water.

\subsection{FibroScan measurements}

Transient elastography was measured by FibroScan (manufactured in Echosens, France). More than 10 detections with a success rate of more than $60 \%$ and a quartile range of less than $30 \%$ of the median elasticity were considered effective. The values related to METAVIR fibrosis stages were classified into mild (F1-F2<9.6 kPa) and advanced fibrosis (F3-F4 $\geq 9.6 \mathrm{kPa})$.

\subsection{Statistical analysis}

The FIB-4 and APRI were determined by the following equations: FIB-4=(age $\times$ AST $[\mathrm{U} / \mathrm{L}]) /(\mathrm{PLT}$ $\left.\left[10^{9} / \mathrm{L}\right] \times \sqrt{\mathrm{ALT}}[\mathrm{U} / \mathrm{L}]\right) ; \mathrm{APRI}=(\mathrm{AST}[\mathrm{U} / \mathrm{L}] /$ upper limit of normal)/(PLT $\left.\left[10^{9} / \mathrm{L}\right] \times 100\right)$. The data with normal distribution were presented as mean values and the measurements of patients with different DARC genotypes were compared using the student's t-test. The rest of the data were presented as median (interquartile range) and compared using the MannWhitney U test.

Moreover, correlations among the routine hematological indices (e.g., liver function tests), the FibroScan measurements, and the HCV RNA titers were analyzed using the Spearman correlation test. It should be mentioned that a p-value of less than 0.05 was considered statistically significant and all the statistical analyses were performed in SPSS software (version 21.0).

\section{Results}

\subsection{DARC polymorphism}

Based on the findings, wild type homozygous genotype $\mathrm{FY}^{*} \mathrm{~A} / \mathrm{FY}^{*} \mathrm{~A}$ was most predominant $(\mathrm{n}=211$, $86.1 \%$ ), while heterozygous genotype $\mathrm{FY}^{*} \mathrm{~A} / \mathrm{FY}^{*} \mathrm{~B}$ was the least common $(n=34,13.9 \%)$. Moreover, genotype $\mathrm{FY}^{*} \mathrm{~B} / \mathrm{FY}^{*} \mathrm{~B}$ was absent in these patients. The distribution of the observed genotypes was in agreement with the Hardy-Weinberg equilibrium $(\mathrm{P}=0.24)$.

\subsection{Relationship of DARC polymorphism with} Fibroscan measurement results and serum biochemical

Regarding the demographic characteristics and routine blood and liver function test results of the wild type homozygous and heterozygous genotype patients (Table 1), there was no significant difference between the genotypes except for ALT $(\mathrm{P}=0.025)$ and the AST/ALT ratio $(\mathrm{P}=0.004)$. The log-transformed HCV RNA titers seen in the wild type homozygous

Table 1. Demographic characteristics, and routine blood and liver function test results of chronic HCV-infected patients

\begin{tabular}{|c|c|c|c|c|}
\hline & \multirow{2}{*}{ HCV patients } & \multicolumn{2}{|c|}{ DARC genotyping } & \multirow{2}{*}{$\mathbf{P}$} \\
\hline & & $\mathbf{F Y} * \mathbf{A} / \mathbf{F Y} * \mathbf{A}$ & $\mathbf{F Y} * \mathbf{A} / \mathbf{F Y} * \mathbf{B}$ & \\
\hline $\mathbf{n}$ & 245 & 211 & 34 & \\
\hline Age & $53.7 \pm 13.7$ & $54.6 \pm 13.8$ & $48.2 \pm 11.8$ & \\
\hline Gender (M/F) & $128 / 117$ & $109 / 103$ & $19 / 14$ & \\
\hline $\operatorname{RBC}\left(10^{12} / \mathrm{L}\right)$ & $4.5 \pm 0.6$ & $4.5 \pm 0.6$ & $4.4 \pm 0.8$ & 0.438 \\
\hline HGB (g/L) & $137.9 \pm 18.7$ & $138.1 \pm 18.0$ & $136.8 \pm 23.1$ & 0.757 \\
\hline WBC $\left(10^{9} / \mathrm{L}\right)$ & $4.3(3.5 \sim 5.4)$ & $4.3(3.5 \sim 5.5)$ & $4.2(3.3 \sim 5.3)$ & 0.298 \\
\hline Neutrophil $\left(10^{9} / \mathrm{L}\right)$ & $2.20(1.66 \sim 3.08)$ & $2.19(1.71 \sim 3.16)$ & $2.20(1.45 \sim 2.65)$ & 0.204 \\
\hline Lymphocyte $\left(10^{9} / \mathrm{L}\right)$ & $1.67(1.35 \sim 2.21)$ & $1.67(1.38 \sim 2.21)$ & $1.73(1.09 \sim 2.21)$ & 0.566 \\
\hline Monocyte $\left(10^{9} / \mathrm{L}\right)$ & $0.19(0.14 \sim 0.25)$ & $0.19(0.14 \sim 0.25)$ & $0.15(0.12 \sim 0.23)$ & 0.056 \\
\hline Eosinophil $\left(10^{9} / \mathrm{L}\right)$ & $0.07(0.04 \sim 0.11)$ & $0.07(0.04 \sim 0.12)$ & $0.07(0.03 \sim 0.09)$ & 0.403 \\
\hline Basophil $\left(10^{9} / \mathrm{L}\right)$ & $0.01(0 \sim 0.01)$ & $0.01(0 \sim 0.01)$ & $0.01(0 \sim 0.01)$ & 0.397 \\
\hline Platelet $\left(10^{9} / \mathrm{L}\right)$ & $156.7 \pm 64.0$ & $156.5 \pm 65.6$ & $158.0 \pm 53.1$ & 0.900 \\
\hline ALT (U/L) & $123.5(85.0 \sim 163.3)$ & $119.0(82.7 \sim 155.9)$ & $135.4(110.7 \sim 195.9)$ & 0.025 \\
\hline $\operatorname{AST}(\mathrm{U} / \mathrm{L})$ & $79.8(55.7 \sim 109.5)$ & $79.8(55.4 \sim 108.3)$ & $80.5(56.2 \sim 112.8)$ & 0.771 \\
\hline ALB (g/L) & $43.8 \pm 5.2$ & $43.7 \pm 5.0$ & $44.0 \pm 6.1$ & 0.728 \\
\hline AST/ALT & $0.66(0.53 \sim 0.85)$ & $0.67(0.54 \sim 0.86)$ & $0.58(0.42 \sim 0.69)$ & 0.004 \\
\hline APRI & $1.31(0.84 \sim 2.31)$ & $1.27(0.84 \sim 2.29)$ & $1.47(0.86 \sim 2.44)$ & 0.770 \\
\hline FIB-4 & $2.67(1.62 \sim 4.32)$ & $2.71(1.63 \sim 4.74)$ & $2.53(1.15 \sim 3.38)$ & 0.146 \\
\hline
\end{tabular}

HCV: hepatitis C virus; DARC: Duffy antigen/chemokines receptor; RBC: red blood count; HGB: hemoglobin; WBC: white blood count; ALT: alanine aminotransferase; AST: aspartate aminotransferase; ALB: albumin; APRI: AST to platelet ratio index; FIB-4: Fibrosis 4 score 


\begin{tabular}{|c|c|c|c|c|}
\hline \multirow{2}{*}{ Log (RNA copies/mL) } & \multirow{2}{*}{ HCV patients } & \multicolumn{2}{|c|}{ DARC genotyping } & \multirow{2}{*}{$\mathbf{P}$} \\
\hline & & FY*A/FY*A & FY*A/FY*B & \\
\hline Total & $6.93(6.03 \sim 7.56)$ & $6.95(6.13 \sim 7.56)$ & $6.35(5.48 \sim 7.54)$ & 0.241 \\
\hline$\leq 6$ & $4.92(4.51 \sim 5.56)$ & $4.87(4.49 \sim 5.52)$ & $5.45(4.79 \sim 5.80)$ & 0.122 \\
\hline$>6$ & $7.25(6.69 \sim 7.75)$ & $7.24(6.68 \sim 7.75)$ & $7.54(7.04 \sim 7.69)$ & 0.331 \\
\hline
\end{tabular}

\begin{tabular}{|c|c|c|c|c|}
\hline \multirow{2}{*}{ FibroScan } & \multirow{2}{*}{ HCV patients } & \multicolumn{2}{|c|}{ DARC genotyping } & \multirow{2}{*}{$\mathbf{P}$} \\
\hline & & $\mathbf{F Y}{ }^{*} \mathbf{A} / \mathbf{F Y}^{*} \mathbf{A}$ & $\mathbf{F Y} * \mathbf{A} / \mathbf{F Y} \mathbf{H}^{*} \mathbf{B}$ & \\
\hline Total & $8.9(6.1 \sim 13.9)$ & $8.9(6.2 \sim 14.0)$ & $8.3(5.1 \sim 10.5)$ & 0.325 \\
\hline F1, F2 & $6.3(5.0 \sim 7.8)$ & $6.3(5.2 \sim 7.8)$ & $5.1(5.0 \sim 7.7)$ & 0.531 \\
\hline F3, F4 & $14.3(11.4 \sim 24.5)$ & $14.9(11.5 \sim 27.5)$ & $11.5(10.1 \sim 17.3)$ & 0.242 \\
\hline
\end{tabular}

HCV: hepatitis C virus; DARC: Duffy antigen/ chemokines receptor

and heterozygous genotype patient groups were also measured and compared (Table 2); however, there was no significant difference between them.

Moreover, no significant difference was observed between the genotype groups when they were further subdivided into the log (RNA copies/mL) $\leq 6$ and $\log$ (RNA copies $/ \mathrm{mL}$ ) $>6$. Finally, the results of FibroScan measurements of HCV-infected patients were recorded and summarized in Table 3. There was no significant difference between the wild type homozygous and heterozygous genotype groups in terms of combined FibroScan measurements. Furthermore, no significant difference was found between the subgroups with mild and advanced fibrosis in this regard.

\subsection{Correlation analyses}

Results of the correlations between the liver function biomarkers, the liver fibrosis indices, and the HCV RNA titers were analyzed (Figure 1). The

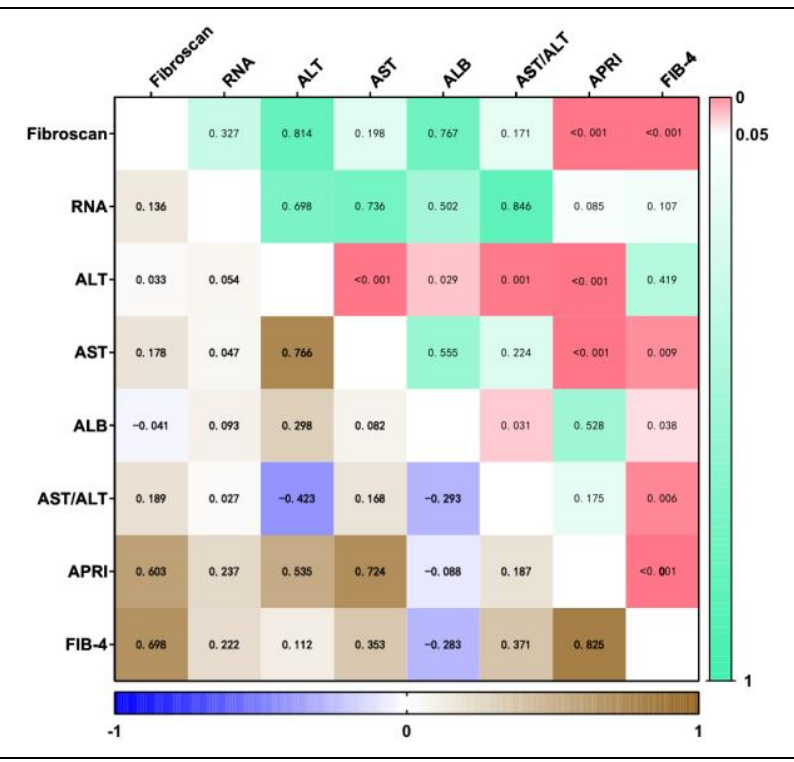

Figure 1. Results of the Spearman's rank correlations among the clinical characteristics. The upper and lower triangles represent the p- and rho values. ALT: alanine aminotransferase; AST: aspartate aminotransferase; ALB: albumin; APRI: AST to platelet ratio index; FIB-4: Fibrosis 4 score collected data indicated that APRI and FIB-4 were correlated positively with FibroScan measurements; however, this was not the case with AST/ALT. FibroScan measurements, HCV RNA titers, and liver function biomarkers (ALT, AST, and ALB) did not correlate significantly with each other.

\section{Discussion}

According to the World Health Organization, approximately 71 million individuals are chronically infected with HCV globally. In different regions of China, the prevalence of chronic HCV infection ranges from $0.43 \%$ to $3.2 \%$ (14). Therefore, it is necessary to study the mechanism of HCV to reduce the risk of disease progression.

In this study, the associations of DARC polymorphism with Fibroscan measurement results and serum biochemical were analyzed and it was found that ALT concentrations and the AST/ALT ratio differed significantly between patients with the wild type homozygous and heterozygous genotypes of DARC.

The ALT is a cytosolic liver enzyme that leaks into the circulation during hepatocyte necrosis. Therefore, ALT activity is commonly measured to assess liver disease severity and, in theory, provides a good marker of liver injury. Moreover, ALT variation is also attributed to immunogenetic factors (15) with the heritability of ALT fluctuations ranging from $35-61 \%$ (16).

Numerous studies support this idea; for instance, Larrieta-Carrasco et al. (17) investigated 288 singlenucleotide polymorphisms (SNPs) in genome-wide association studies and found that several SNPs of certain genes were significantly associated with elevated ALT levels in Mexican adults with an admixed ancestry. Moreover, Viitasalo et al. (15) showed that children carrying the $\mathrm{T}$ allele of the MBOAT7 polymorphism had higher plasma ALT than the noncarriers. In addition, a meta-analysis indicated that the PNPLA3 rs738409 polymorphism can have a differentiated influence on the ALT level (16).

The present study is the first one to identify the 
association between the DARC rs12075 and ALT concentrations in chronic HCV infected patients. The mechanism is unclear; nevertheless, we considered that the circulating levels of proinflammatory chemokines can be regulated by DARC polymorphism which also contributes to liver inflammation and damage during chronic HCV infection. $\mathrm{FY}^{*} \mathrm{~A}$ allele is a potentially valuable protective factor against hepatocyte damage-induced ALT leakage in chronic HCV infected patients. This provides new evidence to DARC which would be helpful for future risk assessment and personalized treatment of chronic HCV infection.

Besides ALT and AST/ALT, no other indices were found to be associated with DARC polymorphism. APRI and FIB-4 indices are based on routinely available clinical laboratory parameters and have significant accuracy in the diagnosis of liver fibrosis $(18,19)$. Moreover, the FibroScan has been shown to accurately predict histological fibrosis and its guidelines recommend its use for the determination of the fibrosis stage in HCV positive patients (20-22).

These negative associations of DARC rs12075 polymorphism with APRI, FIB-4, and FibroScan results suggest that genetic factors that determine the circulating levels of chemokines and ALT are not necessarily risk factors for the progression of liver fibrosis in chronic HCV-infected patients. This result is concordant with those of previous research that used non-invasive serum liver-related markers (23) and liver biopsy (24).

However, the findings of a study conducted by Jiménez-Sousa et al. (25) were inconsistent. In their retrospective cohort study (based on a repeated measures design) performed on 208 chronic HCVinfected patients, rs12075 was correlated with the liver fibrosis progression in HCV-infected patients. Therefore, further research with larger sample sizes in various populations is required. The Rs2814778, a specific SNP at the DARC gene promoter, results in a DARC-null phenotype and has been found to be a predictor of low counts of leukocyte and neutrophil $(26,27)$. However, in this study, it was found that DARC polymorphism (rs12075) was not significantly associated with white blood counts in HCV positive patients and it is suggested that these two SNPs have different biological functions.

In the correlation analyses, FibroScan was correlated positively with APRI (rho $=0.603$, $\mathrm{P}=1.379 \times 10^{-6}$ ) and FIB-4 (rho=0.698, $\mathrm{P}=4.522 \times 10^{-9}$ ). Based on the results of the present research, APRI and FIB-4 are useful liver fibrosis parameters in chronic HCV infection which is consistent with those of other studies $(28,29)$.

The findings are conflicting regarding the merit of using AST/ALT as a fibrosis marker since the study results range from showing no value to demonstrating that this ratio can be a good predictor of liver fibrosis (30). According to the results of this study, FibroScan measurements did not significantly correlate with AST/ALT, indicating that this ratio is not a good and discriminative index for estimating fibrosis extent in chronic HCV infection as previously demonstrated by others $(30,31)$. Al-Khurri et al. in their study concluded that serum HCV RNA level does not determine the development of liver damage and fibrosis (32). This is in line with the results of this study and suggests that HCV RNA titers and liver injury markers are not effective in the diagnosis of the degree of liver fibrosis in chronic HCV positive patients.

\section{Conclusion}

Based on the results, it can be concluded that in the Han population of Dalian, DARC polymorphism can affect ALT concentrations and that the FY*A allele may be a valuable protective factor against hepatocyte damage and associated ALT leakage in chronic HCV-infected patients. However, DARC polymorphism had no significant associations with HCV RNA titers or liver fibrosis in chronic HCVinfected patients.

Moreover, according to the correlation analyses, APRI and FIB-4, unlike AST/ALT, were useful parameters of liver fibrosis in chronic hepatitis $C$. The HCV RNA titers and liver injury markers were not useful in the estimation of the fibrosis stage in chronic hepatitis C patients. Nevertheless, the statistic population of this study was relatively small, therefore, it is recommended to conduct further research with larger sample sizes in various populations.

\section{References}

1. Cosset FL, Mialon C, Boson B, Granier C, Denolly S. HCV interplay with lipoproteins: inside or outside the cells? Viruses. 2020; 12(4):434. doi:10.3390/v12040434. [PubMed: 32290553].

2. Karsdal MA, Daniels SJ, Holm Nielsen S, Bager C, Rasmussen DGK, Loomba $\mathrm{R}$, et al. Collagen biology and non-invasive biomarkers of liver fibrosis. Liver Int. 2020;40(4):736-50. doi: 10.1111/liv.14390. [PubMed: 31997561].

3. Höher G, Rodrigues MMO, Waskow G, Agnes G, Von Burg PV, Onsten $\mathrm{T}$, et al. Identification of ACKR1 variants associated with altered Duffy phenotype expression in blood donors from southern Brazil. Transfus Apher Sci. 2020;59(4)102768. doi: 10.1016/j.transci.2020.102768. [PubMed: 32276863]

4. Morein D, Erlichman N, Ben-Baruch A. Beyond cell motility: the expanding roles of chemokines and their receptors in malignancy. Front Immunol. 2020;11:952. doi: 10.3389/ fimmu.2020.00952. [PubMed: 32582148].

5. Jenkins BD, Martini RN, Hire R, Brown A, Bennett B, Brown I, et al. Atypical chemokine receptor 1 (DARC/ACKR1) in breast tumors is associated with survival, circulating chemokines, tumor-infiltrating immune cells, and african ancestry. Cancer Epidemiol Biomarkers Prev. 2019;28(4):690-700. doi: 10.1158/1055-9965.epi-18-0955. [PubMed: 30944146].

6. Höher G, Fiegenbaum M, Almeida S. Molecular basis of the Duffy blood group system. Blood Transfus. 2018;16(1):93-100. doi: 10.2450/2017.0119-16. [PubMed: 28151395].

7. Davis MB, Walens A, Hire R, Mumin K, Brown AM, Ford D, et al. Distinct transcript isoforms of the atypical chemokine receptor 
1 (ACKR1)/Duffy antigen receptor for chemokines (DARC) gene are expressed in lymphoblasts and altered isoform levels are associated with genetic ancestry and the duffy-null allele. PLoS One. 2015;10(10):e0140098. doi: 10.1371/journal.pone. 0140098. [PubMed: 26473357].

8. Kano FS, de Souza AM, de Menezes Torres L, Costa MA, SouzaSilva FA, Sanchez BAM, et al. susceptibility to plasmodium vivax malaria associated with darc (Duffy antigen) polymorphisms is influenced by the time of exposure to malaria. Sci Rep. 2018;8(1):13851. doi: 10.1038/s41598-01832254-z. [PubMed: 30218021].

9. Namdar A, Dunsmore G, Shahbaz S, Koleva P, Xu L, Jovel J, et al. CD71(+) erythroid cells exacerbate HIV-1 susceptibility, mediate trans-infection, and harbor infective viral particles. mBio. 2019;10(6) :e02767-19. doi: 10.1128/mBio.02767-19. [PubMed: 31772057].

10. Li DD, Yang C, Shao ZM, Yu KD. Effect of functional genetic variants in chemokine decoy receptors on the recurrence risk of breast cancer. Cancer Med. 2018;7(11):5497-504. doi: 10.1002/cam4.1823. [PubMed: 30358125].

11. Martins ML, da Silva AR, Santos HC, Alves MT, Schmidt LC, Vertchenko SB, et al. Duffy blood group system: New genotyping method and distribution in a Brazilian extraAmazonian population. Mol Cell Probes. 2017;35:20-6. doi: 10.1016/j.mcp.2017.06.001. [PubMed: 28587995].

12. Chaudhry S, Emond J, Griesemer A. Immune cell trafficking to the liver. Transplantation. 2019;103(7):1323-37. doi: 10.1097/tp.0000000000002690. [PubMed: 30817405].

13. Chalin A, Lefevre B, Devisme C, Barget N, Amiot L, Samson M. Circulating levels of CXCL11 and CXCL12 are biomarkers of cirrhosis in patients with chronic hepatitis C infection. Cytokine. 2019;117:72-8. doi: 10.1016/j.cyto.2019.02.006. [PubMed: 30826602].

14. Zhang ST, Shi M, Shao LN, Zhou SH, Yu WJ, Chen M, et al. Atypical chemokine receptor 1 polymorphism can not affect susceptibility to hepatitis C virus. Balkan Med J. 2017; 34(4):308-12. doi: 10.4274/balkanmedj.2016.0766. [PubMed: 28443566].

15. Viitasalo A, Eloranta AM, Atalay M, Romeo S, Pihlajamäki J, Lakka TA. Association of MBOAT7 gene variant with plasma ALT levels in children: the PANIC study. Pediatr Res. 2016;80(5):651-5. doi: 10.1038/pr.2016.139. [PubMed: 27411039].

16. Liu Z, Ning H, He X, Que S, Zhou L. Meta-analysis reveals a specific association of the PNPLA3 I148M polymorphism with ALT level in adolescents. Per Med. 2015;12(2):67-82. doi: 10.2217/pme.14.72. [PubMed: 29754542].

17. Larrieta-Carrasco E, Flores YN, Macías-Kauffer LR, RamírezPalacios P, Quiterio M, Ramírez-Salazar EG, et al. Genetic variants in COL13A1, ADIPOQ and SAMM50, in addition to the PNPLA3 gene, confer susceptibility to elevated transaminase levels in an admixed Mexican population. Exp Mol Pathol. 2018;104(1):50-8. doi: 10.1016/j.yexmp.2018.01.001. [PubMed: 29307798].

18. Gounder PP, Haering C, Bruden DJT, Townshend-Bulson L, Simons BC, Spradling PR, et al. Does incorporating change in APRI or FIB-4 indices over time improve the accuracy of a single index for identifying liver fibrosis in persons with chronic hepatitis c virus infection? J Clin Gastroenterol. 2018;52(1):60-6. doi: 10.1097/mcg.0000000000000753. [PubMed: 27875352].

19. Tseng CH, Chang CY, Mo LR, Lin JT, Tai CM, Perng DS, et al. Acoustic radiation force impulse elastography with Apri and FIB-4 to identify significant liver fibrosis in chronic hepatitis B patients. Ann Hepatol. 2018;17(5):789-94. doi: 10.5604/01.3001.0012.3137. [PubMed: 30145564].

20. Lim JK, Flamm SL, Singh S, Falck-Ytter YT. American gastroenterological association institute guideline on the role of elastography in the evaluation of liver fibrosis. Gastroenterology. 2017;152(6):1536-43. doi: 10.1053/j.gastro. 2017.03.017. [PubMed: 28442119].

21. Xiao G, Zhu S, Xiao X, Yan L, Yang J, Wu G. Comparison of laboratory tests, ultrasound, or magnetic resonance elastography to detect fibrosis in patients with nonalcoholic fatty liver disease: A meta-analysis. Hepatology. 2017; 66(5):1486-501. doi: 10.1002/hep.29302. [PubMed: 28586172].

22. Guo L, Zheng L, Hu L, Zhou H, Yu L, Liang W. Transient elastography (fibroscan) performs better than non-invasive markers in assessing liver fibrosis and cirrhosis in autoimmune hepatitis patients. Med Sci Monit. 2017;23:510612. doi: 10.12659/msm.907300. [PubMed: 29073121].

23. Shao LN, Zhang ST, Zhou SH, Yu WJ, Liu M. Atypical chemokine receptor 1 polymorphism cannot be used as an indicator of liver fibrosis progression in Hepatitis C virus positive patients. Pak J Med Sci. 2017;33(5):1134-7. doi: 10.12669/pjms. 335.12590. [PubMed: 29142552].

24. Lettow I, Berres ML, Schmitz P, Muller T, Berg T, Neumann UP, et al. A Duffy antigen receptor for chemokines (DARC) polymorphism that determines pro-fibrotic chemokine serum concentrations is not directly associated with severity of hepatitis C infection. Hum Immunol. 2011;72(3):273-7. doi: 10.1016/j.humimm.2010.12.002. [PubMed: 21156192].

25. Jimenez-Sousa MA, Gomez-Moreno AZ, Pineda-Tenor D, Sanchez-Ruano JJ, Artaza-Varasa T, Martin-Vicente M, et al. Impact of DARC rs12075 variants on liver fibrosis progression in patients with chronic hepatitis C: A retrospective study. Biomolecules. 2019;9(4):143. doi: 10.3390/biom9040143. [PubMed: 30970632].

26. Rappoport N, Simon AJ, Amariglio N, Rechavi G. The Duffy antigen receptor for chemokines, ACKR1,- 'Jeanne DARC' of benign neutropenia. Br J Haematol. 2019;184(4):497-507. doi: 10.1111/bjh.15730. [PubMed: 30592023].

27. Fragiadaki I, Papadakis S, Sevastaki G, Sfyridaki K, Mavroudi I, Goulielmos GN, et al. Increased frequency of the single nucleotide polymorphism of the DARC/ACKR1 gene associated with ethnic neutropenia in a cohort of European patients with chronic idiopathic neutropenia. Am J Hematol. 2020; 95(7):E163-6. doi: 10.1002/ajh.25813. [PubMed: 32243614].

28. Yosry A, Fouad R, Alem SA, Elsharkawy A, El-Sayed M, Asem N, et al. FibroScan, APRI, FIB4, and GUCI: Role in prediction of fibrosis and response to therapy in Egyptian patients with HCV infection. Arab J Gastroenterol. 2016;17(2):78-83. doi: 10.1016/j.ajg.2016.05.002. [PubMed: 27353055].

29. Cheng CH, Chu CY, Chen HL, Lin IT, Wu CH, Lee YK, et al. Subgroup analysis of the predictive ability of aspartate aminotransferase to platelet ratio index (APRI) and fibrosis-4 (FIB-4) for assessing hepatic fibrosis among patients with chronic hepatitis C. J Microbiol Immunol Infect. 2020;53(4):542-9. doi: 10.1016/j.jmii.2019.09.002. [PubMed: 31831303].

30. Guéchot J, Boisson RC, Zarski JP, Sturm N, Calès P, Lasnier E. AST/ALT ratio is not an index of liver fibrosis in chronic hepatitis $\mathrm{C}$ when aminotransferase activities are determinate according to the international recommendations. Clin Res Hepatol Gastroenterol. 2013;37(5):467-72. doi: 10.1016/j. clinre.2013.07.003. [PubMed: 23932706].

31. Eminler AT, Ayyildiz T, Irak K, Kiyici M, Gurel S, Dolar E, et al. AST/ALT ratio is not useful in predicting the degree of fibrosis in chronic viral hepatitis patients. Eur J Gastroenterol Hepatol. 2015;27(12):1361-6. doi: 10.1097/meg.0000000000000468. [PubMed: 26352130].

32. Al-Khurri LE, Al-Khafaji KR, Al-Salihi SA, Alwaysi SA, AlAkayshi RJ. Serum HCV-RNA levels in patients with chronic hepatitis C: Correlation with histological features. Arab J Gastroenterol. 2009;10(1):10-3. doi: 10.1016/j.ajg.2009. 03.003. [PubMed: 24842130]. 